\title{
Identification of Axial Forces on Statically Indeterminate Pin-Jointed Trusses by a Nondestructive Mechanical Test
}

\author{
Emilio Turco*
}

DADU, Università degli Studi di Sassari, 07041 Alghero, Sassari, Italia

\begin{abstract}
This work concerns the stress-state identification, produced by loads or dislocations, of statically indeterminate structures. In particular, this paper deals with the case of pin-jointed trusses, since they are a combination of simplicity of the structural model with very interesting technical applications. We present an approach that follows the main guidelines of the flexibility method to relate an additional load and the consequent nodal displacements. When a sufficient number of nodal displacements are measured, they produce a system of equations that when solved furnishes a complete reconstruction of the stress-state to identify. In order to highlight the potentialities and the limits of the proposed approach and also to delineate its main characteristics, some simple tests are discussed and analyzed.
\end{abstract}

Keywords: Inverse problems, stress-state identification, nondestructive tests.

\section{INTRODUCTION}

Knowledge of the stress-state of a mechanical part, such as an engine, a wheel or a gear, is significant for the mechanical industry since the excess of stresses could give rise to a premature and sudden break. In order to design possible reiforcements and risk assessments, an in-depth knowledge of the actual stress-state on a structure is necessary in the field of civil engineering. Another research field where the identification of the actual stress has some relevant applications is biomechanics: an enlightening example is related to the determination of artery hypertension, as reported by [1]. These brief discussions contribute in highlighting the importance of these issues in the scientific community.

It is known that a complete estimation of the stress-state is required for residual stresses, i.e. stresses corresponding to null loads [2]. In the technical literature, various approaches have been reported in relation to the problem of identifying the actual state of stress in a structure. These methods can be subdivided into destructive and nondestructive tests. The first class of methods includes all the tests requiring an hole or a cut on the selected part. This causes a relaxation of the stress field and consequent displacements; the measurement of such displacements gives the data required to reconstruct the pre-existing stress field. In fact, it is enough to impose the opposite of the measured displacement field and derive the consequent stress field, for example the papers of [3] and [4]. Destructive tests obviously have the disadvantage to partially or totally compromise the element to test.

On the other hand, nondestructive tests to some extent deduce the stress field by measuring the effects induced by a prescribed signal. Among others, the method based on the

*Address correspondence to this author at the DADU, Università degli Studi di Sassari, 07041 Alghero, Sassari, Italia; Tel: +39 079 9720408;

Fax: +39079 9720420; E-mail: emilio.turco@ uniss.it
$\mathrm{X}$-ray diffraction is quite widespread but limited by the poor capacity of penetration. Another well-established approach is the ultrasonic one, based upon the dependence between the wave velocity and stresses. For both these methods, the book of [5] contains a brief introduction and several references. Also the article of [6] introduces the use of magnetophotoelasticity in this same framework.

Both in the static and dynamic fields signals and measurements of mechanical type, forces and displacements, have been considered, both in static and dynamic field. In the technical literature [2] is an attempt to frame the problem furnishing some useful and fairly general guidelines [7]. shows that residual stresses are defined on the boundary when the Dirichlet to Neumann map is known [8]. gives some useful comments on the modeling of the residual stress suggesting the use of a model based on the Hartig's law. These suggestions are also partially described in [9], which improves the model already presented in [7] showing the iniectivity of the map. Another interesting work contained in [10] concerns the identification of pre-stressed cable structures by dynamic tests.

This work has initially portrayed a nondestructive stressstate identification strategy applicable to real structural problems. The work provide details about simplicity and technical application of the pin-jointed trusses, which allow to explore the essential aspects of the problem without involving heavy computations. However, the formulation developed for pin-jointed trusses can be easily extended to any problem discretized by finite elements by using the matrix language.

The paper shows the significance of the relation between an additional static load and the consequent displacements on the examined structure. Moving into the framework of the force method, this relation is written using standard elasticity hypotheses such as Hooke's law and small displacements. 
Some brief consideration relative to the use of the Hartig's law are also presented. Here, the choices in the design of the proposed algorithm were simple in order to be easily implemented on an actual problem. This, for example, was the motivation to use the additional static loads with respect to those dynamical.

After this brief introduction, Section 2 contains a brief discussion on the statement of the underlying identification problem and a discussion on the main tools used to build the identification strategy for statically indeterminate pin-jointed trusses. Also some remarks on the possible use of Hartig's law are briefly sketched. Section 3 comprises of some results on simple pin-jointed truss structures useful to illustrate the main characteristics of the proposed approach. Concluding remarks are elaborated in Section 4.

\section{IDENTIFICATION PROBLEM: STATEMENT AND TOOLS}

\subsection{Problem Sketch}

It is considered an assigned configuration of a pin-jointed truss, denoted as current, the structure is also considered in equilibrium under the external loads. Axial forces on the truss are collected in the vector $\mathbf{t}$ and can be produced by loads and dislocations such as thermal variation or lack of fit between the bars. The identification of vector $\mathbf{t}$ should be done by a simple procedure possibly adaptable to in situ tests. In case the system is statically determined the problem can be solved through the solution of equilibrium equations, obviously. Conversely, if the truss is statically undetermined it is necessary an ad hoc identification procedure.

Beginning from the current configuration, an additional load was applied and the truss moves to a new configuration denoted as test configuration. The increment of axial forces and of nodal displacements were denoted by $\dot{\boldsymbol{t}}$ and $\dot{\boldsymbol{u}}$ respectively. The question is wheter it is possible to identify the axial force vector $\boldsymbol{t}$ by assuming the adequate number of the displacement increments of the vector $\dot{\boldsymbol{u}}$ as known? It has been assumed that identification problem can be treated as linear and elastic by considering the position of each node in the current configuration, and so the length of each bar, the material constant, such as the Young's modulus, and, finally, the cross-section area of each bar as known.

There are, basically, two alternative ways to solve the identification problem as mentioned earlier. The first follows the guidelines outlined by the stiffness method that substantially assumes the nodal displacements as primary unknows. On the other hand, the second method is the flexibility method which assumes the axial forces on the truss as primary unknowns. Here, the unknowns factors are axial forces and so the preferences will be given to the second method. The potentialities of the flexibility method was explored to build an algorithm able to solve our problem. In the next section, the identification problem will be defined in a more formal way by building a tool which will generate the system of equations and solving those equations the axial force vector $\boldsymbol{t}$ will be obtained.

\subsection{Tools}

Following the way traced in [11] and more recently in [12], an assembly of $b$ bars and $j$ joints on which $c$ kinematic constraints prevent some displacements has been considered. External forces acting on uncostrained joints are collected in the $(d j-c)$-dimensional vector $\boldsymbol{l}$ where $d$ is equal to 2 or 3 for two- and three-dimensional problem respectively. The equilibrium matrix $\boldsymbol{A}$ links the axial forces on the bars collected in the $b$-dimensional vector $\boldsymbol{t}$ with the load vector $\boldsymbol{l}$ At $=\boldsymbol{l}$

and, consequently, has dimensions $(d j-c) \times b$.

Kinematic compatibility is ensured by the matrix $\boldsymbol{B}=$ $\boldsymbol{A}^{T}$, see [13], which links the $(d j-c)$-dimensional vector $\boldsymbol{u}$ of nodal displacements to the $b$-dimensional vector $\boldsymbol{e}$ binding bar elongations

$B \boldsymbol{u}=\boldsymbol{e}$

The relation between bar elongations $\mathbf{e}$ and axial forces $\mathbf{t}$ can be written by means of the axial flexibility matrix $\mathbf{F}$ and the vector $\overline{\boldsymbol{e}}$ of the imposed bar elongation collects bar elongations deriving for example from thermal variation or lack of fit between bars. We can write this constitutive relation as $\boldsymbol{e}=\boldsymbol{F t}+\overline{\boldsymbol{e}}$

Flexibility matrix is square, with $b$-dimension, and diagonal with the $i$-th term, in the case we use Hooke's law, which is equal to

$F_{i}=\frac{\ell_{i}}{E_{i} A_{i}}$

and depends on the length $\ell_{i}$, the cross-section area $A_{i}$ and the Young's modulus $E_{i}$ of the $i$-th bar. It has been emphasized that the above relation is deduced from the St. Venant's problem and therefore is referred to the initial and stress-free configuration.

Here the case of statically indeterminate and kinematiccally determined pin-jointed trusses as defined in [11] are considered. A structure is supposed to belong to this class if the equilibrium system of equations has infinite solutions for any right hand side for the equilibrium system (1), while the compatibility system of equations (2) has a unique solution for some particular right hand side, but otherwise has no solution. This choice derives from the technical importance of this class of structures. Systems of equations (1), (2) and (3) can be arranged in various ways to find the unknown vector $\boldsymbol{u}, \boldsymbol{e}$ and $\boldsymbol{t}$ of the so-called direct problem, giving the wellknown solution algorithm, such as the force method and the displacement method.

For the stress state identification problem defined above the force method appears to be the most promising. The road traced by the force method works by assuming the bar axial forces as primarily unknowns. This method can be synthesized starting from the representation of bar axial forces which satisfy, a priori, the equilibrium equations:

$\boldsymbol{t}=\boldsymbol{t}^{\prime}+\boldsymbol{S x}$

where $\boldsymbol{t}^{\prime}$ solves the system of equations $\boldsymbol{A} \boldsymbol{t}^{\prime}=\boldsymbol{l}$ and the $k$-th column of $\boldsymbol{S}$, that is $\boldsymbol{s}_{\boldsymbol{k}}$, solves the homogeneous system of equations $\boldsymbol{A} \boldsymbol{s}_{\boldsymbol{k}}=\mathbf{0}$. Using the constitutive equations (3), the bar elongation vector can be expressed as

$\boldsymbol{e}=\boldsymbol{F}\left(\boldsymbol{t}^{\prime}+\boldsymbol{S} \boldsymbol{x}\right)+\overline{\boldsymbol{e}}$ 
and, finally, the imposition of the kinematic compatibility in the form proposed in [11]

$S^{T} e=0$

produces the final system of equations

$S^{T} F S x=-S^{T}\left(F t^{\prime}+\bar{e}\right)$

The same result can be obtained by imposing the kinematic compatibility through the use of the virtual work theorem, precisely the virtual force theorem, see for example [13]. The theorem of virtual forces can also be used to evaluate nodal displacements by using the relation

$\tilde{\boldsymbol{l}}^{T} \boldsymbol{u}=\tilde{\boldsymbol{t}}^{T}(\boldsymbol{F t}+\overline{\boldsymbol{e}})$

where $\boldsymbol{t}$ and $\boldsymbol{u}$ are, respectively, the axial force vector and the nodal displacement vector deriving from the solution of (8), while $\tilde{\boldsymbol{t}}$ is a solution of the problem $\boldsymbol{A} \tilde{\boldsymbol{t}}=\tilde{\boldsymbol{l}}$. It is enough to choose as $\tilde{\boldsymbol{l}}$ as a vector with the only nonzero component equal to unity in correspondence with the displacement to calculate and obtain the formula

$u_{k}=\tilde{\boldsymbol{t}}^{T}(\boldsymbol{F} \boldsymbol{t}+\overline{\boldsymbol{e}})$

where $\tilde{\boldsymbol{t}}$ corresponds through the equilibrium matrix, to the particular choice of $\tilde{\boldsymbol{l}}$. Writing (10) for two distinct solutions of (8) labeled with 1 and 2 we obtain

$\dot{u}_{k}=\tilde{\boldsymbol{t}}^{T} \boldsymbol{F} \dot{\boldsymbol{t}}$

where to slim the notation we have used the positions $\dot{t}=t_{2}-t_{1}$ and $\dot{u}=u_{2}-u_{1}$.

In the $i$-th diagonal term of the matrix $\boldsymbol{F}$, see (4), there is the initial bar length that is unknown in our problem while the current bar length is known. For a generic bar and two distinct configuration 1 and 2, the elongation increment is worth $\dot{e}:=\ell_{2}-\ell_{1}=\frac{\ell}{E A} \dot{t}$

while the bar length referred to state 1 is

$\ell_{1}=\ell+e_{1}=\ell\left(1+\frac{t_{1}}{E A}\right)$

Combining the last two relations we obtain

$e=\frac{\ell_{1}}{E A+t_{1}} \dot{t}$

and so the corresponding diagonal term of the flexibility matrix referred to the current configuration is

$\bar{F}_{l}=\left(\frac{\ell_{1}}{E A+t_{1}}\right)_{i}$

Finally, nodal displacement increments collected in the vector $\dot{\boldsymbol{u}}$ can be written as

$\dot{\boldsymbol{u}}=\widetilde{\boldsymbol{T}} \overline{\boldsymbol{F}} \dot{\boldsymbol{t}}$

where $\widetilde{\boldsymbol{T}}$ is formed simply by binding the $\tilde{\boldsymbol{t}}^{T}$ corresponding to the considered nodal displacements.

With these simple tools can be identified the axial forces in an assigned configuration, for example in the current, which can be reached under the action of loads and imposed elongations. By imposing an additional load on the structure produces both the increment of axial forces $\dot{\boldsymbol{t}}$ and of nodal displacements $\dot{\boldsymbol{u}}$. In general, only a part of this displacement increments is measurable. We denote with $\dot{\boldsymbol{u}}_{m}$ the subset of $\dot{\boldsymbol{u}}$ measured. Axial forces to identify $\boldsymbol{t}$ and their increments $\dot{\boldsymbol{t}}$ produced by the additional load, can be represented in such a way that they satisfy the equilibrium equations:

$\boldsymbol{t}=\boldsymbol{t}^{\prime}+\boldsymbol{S x}$

$$
\dot{\boldsymbol{t}}=\dot{\boldsymbol{t}}^{\prime}+\boldsymbol{S} \dot{\boldsymbol{x}}
$$

where the unknown quantities are collected in $\boldsymbol{x}$ and $\dot{\boldsymbol{x}}$. Relation (16) can be seen as a system of equations containing the unknowns $\boldsymbol{x}$ and $\dot{\boldsymbol{x}}$ while $\dot{\boldsymbol{u}}_{m}$ is the known term.

\subsection{Remarks on the constitutive law}

We have discussed above a simple strategy for the identification of the axial forces in pin-jointed trusses based on standard hypotheses of linear elasticity. Here, as suggested in [8], some possible modifications to the constitutive law will be considered briefly in order to modify (15) in accordance with the Hartig's law instead of the classic Hooke's law.

Hartig's law, see [14], can simply be written as

$E:=\frac{d \sigma}{d \varepsilon}=E_{0}-g \sigma$

where the Young's modulus $E$, defined as incremental ratio between the stress $\sigma$ and the engineering strain $\varepsilon$, depends linearly on $\sigma$ through two constitutive coefficients: $E_{0}$, id est the Young's modulus for $\sigma=0$, and the dimensional coefficient $g$. The stress can explicitly be written as

$\sigma=\frac{E_{0}}{g}\left(1-e^{-g \varepsilon}\right)$

from which the engineering strain can easily be deduced

$\varepsilon=-\frac{1}{g} \ln \left(1-\frac{g \sigma}{E_{0}}\right)$

Considering the above two different states, denoted with 1 and 2, for a generic bar of the truss, simple algebraic manipulations permit to write the elongation increment as

$e=\ell_{2}-\ell_{1}=\frac{\varepsilon_{2}-\varepsilon_{1}}{1+\varepsilon_{1}} \ell_{1}$

and by using (20)

$e=\frac{-\frac{\ell_{1}}{g} \ln \frac{E_{0} A-g\left(t_{1}+t\right)}{E A-g t}}{1-\frac{1}{g} \ln \frac{E_{0} A-g t_{1}}{E_{0} A}}$

where the axial force $t=\sigma A$ and its increment $\dot{t}=t_{2}-t_{1}$ on the considered bar are used. This relation links the elongation increments and axial force increments for the considered bar and consequently furnish the definition of the flexibility coefficient for the bar.

The flexibility coefficient for a generic bar can also be derived by linearizing Hartig's law as reported in [8]. Starting from the linearization

$\sigma_{2}-\sigma_{1} \approx\left(E_{0}-g \sigma_{1}\right)\left(\varepsilon_{2}-\varepsilon_{1}\right)$

the combination of (21) and (20) gives

$\dot{e}=\frac{\ell_{1}}{\left(E_{0} A-g t_{1}\right)\left(1-\frac{1}{g} \ln \frac{E_{0} A-g t_{1}}{E_{0} A}\right)} \dot{t}$

that is the link between $\dot{e}$ and $\dot{t}$ which again defines again the flexibility coefficient of the considered bar. Hartig's law or its linearized version produces only a different evaluation of the flexibility coefficients and its use in a stress-state identification algorithm should be evaluated by comparing costs and benefits.

\section{SOME RESULTS}

Here some results referred to simple pin-jointed trusses are being discussed. The aim is to explore the main features of the proposed identification strategy and to render the principal drawbacks evidently.

In the following, trusses formed by bars with the same Young's modulus $E$ and cross-section area $A$ have been considered. Where necessary for numerical calculations, 
$E=200 \mathrm{GPa}$ and $A=3,79 \mathrm{~cm}^{2}$ corresponding to an iron Lprofile 40 x 5 (UNI 5783-73) have been assumed.

\subsection{First example: Two-Bar Truss}

The current configuration of the two-bar pin-jointed truss sketched in Fig. (1) has been considered as first example.

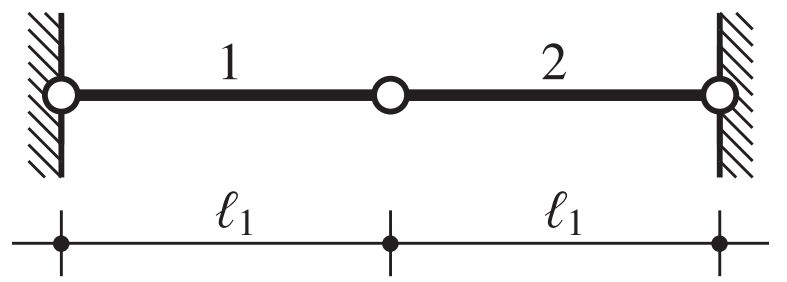

Fig. (1). Two-bars truss problem: geometry of the current configuration.

If the natural or stress-free length of each bar is $\ell=\ell_{1}-$ $e$, on each bar there is an axial force $t=E A e / \ell$. In order to identify the axial forces, starting from the current configuration, a horizontal force $\dot{f}$ on the central node has been applied. The symmetry of the problem produces a horizontal displacement $\dot{u}=\dot{f} \ell / 2 E A$ which characterizes the test configuration.

Assuming the equilibrium of the axial forces in the current configuration, both $\boldsymbol{t}$ and its increment $\dot{\boldsymbol{t}}$, can be represented as

$\boldsymbol{t}=\boldsymbol{S x}$

$$
\dot{\boldsymbol{t}}=\dot{\boldsymbol{t}}^{\prime}+\boldsymbol{S} \dot{\boldsymbol{x}}
$$

since $\boldsymbol{t}^{\prime}=\mathbf{0}$ because there are no external loads in the current configuration. Vectors $\boldsymbol{x}$ and $\dot{\boldsymbol{x}}$ have only one component and represent, for example, the axial force and its increment on the 2 nd bar.

It is simple to prove that the self-stress matrix $\boldsymbol{S}$ is equal to

$\boldsymbol{S}=\left[\begin{array}{l}1 \\ 1\end{array}\right]$

while the vector $\dot{\boldsymbol{t}}^{\prime}$ is worth

$\dot{\boldsymbol{t}}^{\prime}=\left[\begin{array}{l}\dot{f} \\ 0\end{array}\right]$

and, finally, the matrix $\widetilde{\boldsymbol{T}}$ assumes the following form

$\widetilde{\boldsymbol{T}}=\left[\begin{array}{ll}1 & 0\end{array}\right]$

Consequently, the relation between the horizontal displacement increment $\dot{u}$ of the central node and the stressstate, current and its increments, is

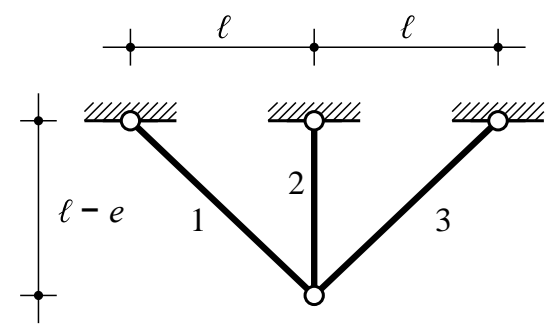

(a)
$\dot{u}=\widetilde{\boldsymbol{T}} \overline{\boldsymbol{F}} \dot{\boldsymbol{t}}=\frac{\ell_{1}}{E A+x}(\dot{f}+\dot{x})$

and can be used to evaluate the unknown $x$ if it is observed that for the symmetry $\dot{x}=-\dot{f} / 2$ and that the incremental displacement $\dot{u}$ is, in our identification problem, a measured quantity and therefore should be treated as known. It results

$x=\frac{1}{2} \frac{\dot{f} \ell_{1}}{\dot{u}}-E A$

which, using the first of (25) to evaluate $t$, produces the same value of the reference solution.

First of all the symmetry condition was used in this case as there was only one measurable displacement. Secondly, an error free solution was obtained since the position of the central node is the same both in the current and natural configurations. In more general examples below, we have the possibility to evaluate the identification error when current and natural configurations are different.

\subsection{Second Example: Three-Bar Truss}

The stress-free configuration of the three-bar truss is illustrated in Fig. (2a).

If an imposed elongation $\bar{e}$ is applied on the 2nd bar, for example deriving from an increment of temperature, this produces a vertical displacement of the free node and axial forces on all the bars. In order to simplify the calculations, we choose for the imposed elongation the value

$\bar{e}=e+\frac{2 e(\ell-e)^{3}}{\left(\sqrt{\ell^{2}+(\ell-e)^{2}}\right)^{3}}$

that produces a vertical displacement of the free-node just equal to $e$ and the axial force vector

$\boldsymbol{t}=\frac{E A e(\ell-e)}{\left(\sqrt{\ell^{2}+(\ell-e)^{2}}\right)^{3}}\left[\begin{array}{c}\sqrt{\ell^{2}+(\ell-e)^{2}} \\ -2(\ell-e) \\ \sqrt{\ell^{2}+(\ell-e)^{2}}\end{array}\right]$

The identification of this axial force vector following the proposed procedure starts from the calculation of the vector $\dot{\boldsymbol{t}}^{\prime}$, of the self-stress matrix $\boldsymbol{S}$ and of the matrix $\widetilde{\boldsymbol{T}}$ Fig. (2b). It is convenient for these calculations to consider the statically determined schemes reported in Fig. (3).

More precisely, $\dot{\boldsymbol{t}}^{\prime}$ derives from the solution of the equilibrium equations related to the scheme reported in Fig. (3a) and results

$\boldsymbol{t}^{\prime}=\frac{1}{\sqrt{2}}\left[\begin{array}{c}\dot{f}_{o}+\dot{f}_{v} \\ 0 \\ -\dot{f}_{o}+\dot{f}_{v}\end{array}\right]$

while $\boldsymbol{S}$ derives from the solution of the equilibrium equa-

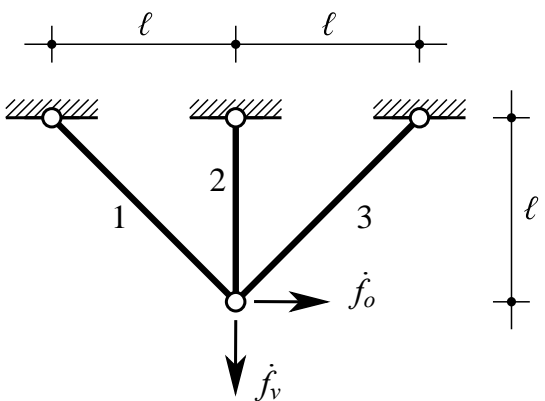

(b)

Fig. (2). Three-bar truss problem: geometry of the stress-free configuration (a) and current configuration with test load (b). 
tions related to the scheme reported in Fig. (3b).

$\boldsymbol{S}=\frac{1}{\sqrt{2}}\left[\begin{array}{l}-1 \\ \sqrt{2} \\ -1\end{array}\right]$

and, finally, the matrix $\widetilde{\boldsymbol{T}}$ can be written by referring to the schemes reported in Fig. (3c) and (3d) and produces the following results

$\widetilde{\boldsymbol{T}}=\frac{\sqrt{2}}{2}\left[\begin{array}{ccc}1 & 0 & -1 \\ 1 & 0 & 1\end{array}\right]$

The displacement increment vector relative to the only free node can be be calculated as

$\dot{\boldsymbol{u}}=\widetilde{\boldsymbol{T}} \overline{\boldsymbol{F}} \dot{\boldsymbol{t}}$

being the term relative to $i$-th bar of the diagonal matrix $\overline{\boldsymbol{F}}$ equal to

$\overline{F_{l}}=\frac{\ell_{i}}{E A-t_{i}}$

The use of axial force representation (17) permits to calculate the explicit expressions of the horizontal $\dot{u}$ and vertical $\dot{v}$ displacement increment of the free node. The result is then

$\dot{u}=\sqrt{2} \frac{\dot{f}_{o} \ell}{E A-\frac{1}{\sqrt{2}} x}$

$$
\dot{v}=\sqrt{2} \frac{\left(\dot{f}_{v}-\dot{x}\right) \ell}{E A-\frac{1}{\sqrt{2}} x}
$$

Assuming that both $\dot{u}$ and $\dot{v}$ are measured and consequently known, we can evaluate the unknowns $x$ and $\dot{x}$ that take on the values

$$
x=\sqrt{2} E A-2 \frac{\dot{f}_{o} \ell}{\dot{u}}
$$

$$
\dot{x}=-\dot{f}_{o} \frac{\dot{v}}{\dot{u}}+\dot{f}_{v}
$$

and using (17) the axial force vector to identify. It follows that

$\boldsymbol{t}_{\mathbf{i d}}=\left[\begin{array}{l}-E A+\sqrt{2} \frac{\dot{f}_{o} \ell}{\dot{u}} \\ \sqrt{2} E A+2 \frac{\dot{f_{o} \ell}}{\dot{u}} \\ -E A+\sqrt{2} \frac{\dot{f_{o}} \ell}{\dot{u}}\end{array}\right]$

where it was noted that for this problem it is necessary to use a horizontal force as test load in order to avoid a null value of horizontal displacement $\dot{u}$.

For the purpose of this work, a reference solution for displacement increments $\dot{u}$ and $\dot{v}$ can be calculated by studying the problem reported in Fig. (2a). Using the stiffness method, the equilibrium imposed on the current configuration, see again Fig. (2a), immediately produces the values

$$
\begin{array}{r}
\dot{u}=\frac{\dot{f}_{o}}{E A} \sqrt{\ell^{2}+(\ell-e)^{2}} \\
\dot{v}=\frac{\dot{f_{v}}}{E A} \frac{\sqrt{\ell^{2}+(\ell-e)^{2}}(\ell-e)}{\sqrt{\ell^{2}+(\ell-e)^{2}}+(\ell-e)}
\end{array}
$$

where the elasticity coefficients $E A / \ell_{i}$ of the $i$-th bar are referred to the stress-free state that in this calculation is considered as known.

Now, the identification error is defined as $\eta=\frac{\left\|t_{\mathrm{id}}-t_{\mathrm{ref}}\right\|}{\left\|t_{\mathrm{ref}}\right\|}$

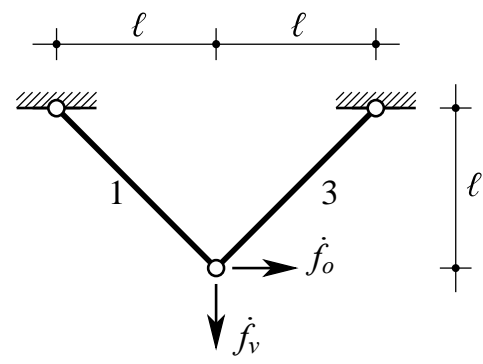

(a)

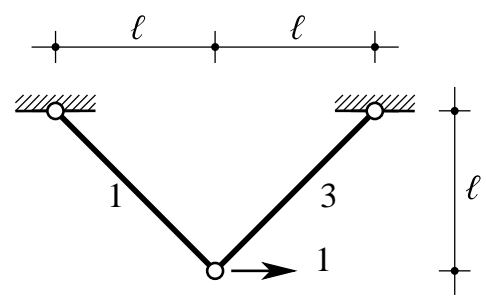

(c)

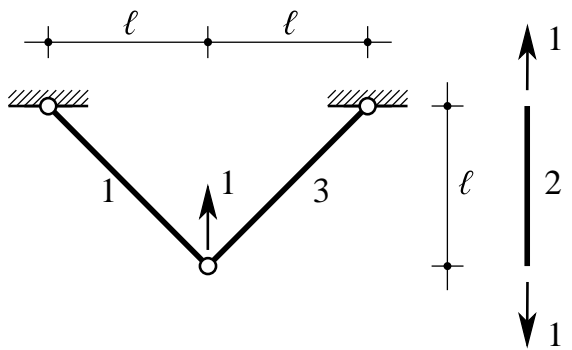

(b)

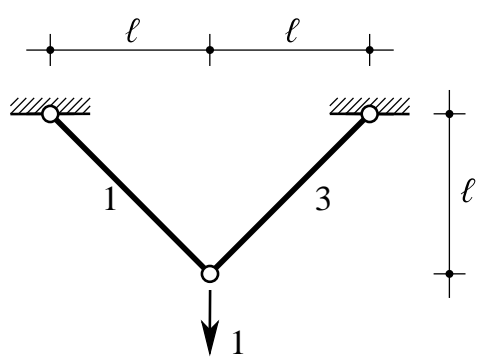

(d)

Fig. (3). Three-bar truss problem: auxiliary schemes for the identification problem.

Table 1. Three-bar Test: Identification Error $\boldsymbol{\eta}$ Varying $\boldsymbol{e} / \boldsymbol{\ell}$ Ratio

\begin{tabular}{|c|c|c|c|c|}
\hline$e / \ell$ & $10^{-6}$ & $10^{-5}$ & $10^{-4}$ & $10^{-3}$ \\
\hline$\eta$ & $5.59 \times 10^{-7}$ & $5.59 \times 10^{-6}$ & $5.59 \times 10^{-5}$ & $5.59 \times 10^{-4}$ \\
\hline
\end{tabular}


where as $\boldsymbol{t}_{\text {ref }}$ is the assumed value of (32), simple calculations show that this error is only dependent on the $e / \ell$ ratio.

Table 1 shows the identification error as the $e / \ell$ ratio increases in the case of $\ell=1 \mathrm{~m}, \dot{f}_{o}=1000 \mathrm{~N}$ and $\dot{f}_{v}=100$ $\mathrm{N}$. It was noted that, in the range considered for the $e / \ell$ ratio, the identification error depends practically on a linear way from $e / \ell$ in such a way that makes the proposed identification strategy accurate.

\subsection{Third Example: Six-Bar Truss}

In the third test we consider the stress-free state of the six-bar pin-joined truss was considered as reported in Fig. (4a). An equilibrated self-stress field can be achieved by increasing temperature $\Delta T$ on the 3 -rd bar. This produces a displacement of the 4-th node, depicted in Fig. (4b), denoted by the parameter $a$.

It is very simple to calculate the displacement parameter $a$ by using the flexibility or the stiffness method. It assumes the value

$a=\frac{1}{1+\sqrt{2}} \alpha_{T} \Delta T \ell$

where $\alpha_{T}$ is the thermic dilatation coefficient characteristic of the considered material. The corresponding vector of axial forces which needs to be identified is equal to

$\boldsymbol{t}=\frac{E A a}{\ell}\left[\begin{array}{c}0 \\ 0 \\ -\sqrt{2} \\ 0 \\ 1 \\ 1\end{array}\right]$

Our identification process uses the forces $f_{o}$ and $f_{v}$ on the 4-th node as a mechanical signal, see Fig. (4c), and as measured displacement increments that do not assume the zero value

$\dot{\boldsymbol{u}}=\left[\begin{array}{c}\dot{u}_{2} \\ \dot{v}_{3} \\ \dot{u}_{4} \\ \dot{v}_{4}\end{array}\right]$

where $u_{k}$ and $v_{k}$ are the horizontal and vertical displacement of the $k$-th node, respectively.

As in the previous examples, both the vector of axial forces $\boldsymbol{t}$ and its increment $\boldsymbol{t}$ can be represented as $\boldsymbol{t}=\boldsymbol{S} \boldsymbol{x}$

$$
\dot{\boldsymbol{t}}=\dot{\boldsymbol{t}}^{\prime}+\boldsymbol{S} \dot{\boldsymbol{x}}
$$

In this case, $\boldsymbol{x}$ and $\dot{\boldsymbol{x}}$ collect the axial forces and their increments produced by the additional forces, $\dot{f}_{o}$ and $\dot{f}_{v}$, on 3 rd and 4th bar. Vector $\dot{\boldsymbol{t}}^{\prime}$ and matrix $\boldsymbol{S}$ can easily be calculated by referring to the schemes reported in Fig. (5a) and Fig. $(\mathbf{5}, \mathbf{b}-\mathbf{c})$ respectively.

Using the parameters $s=a / \sqrt{(\ell+a)^{2}+a^{2}}$ and $c=(\ell+a) / \sqrt{(\ell+a)^{2}+a^{2}}$, they assume the values

$$
\dot{\boldsymbol{t}}^{\prime}=\frac{1}{s^{2}-c^{2}}\left[\begin{array}{c}
\dot{f}_{o} s^{2}-\dot{f}_{v} s c \\
\dot{f_{o}} s c+\dot{f}_{v} s^{2} \\
0 \\
0 \\
\dot{f}_{o} s-\dot{f}_{v} c \\
-\dot{f}_{o} c+\dot{f}_{v} s
\end{array}\right]
$$

$$
\boldsymbol{S}=\left[\begin{array}{cc}
\frac{-\sqrt{2} s}{2(s+c)} & -\frac{\sqrt{2}}{2} \\
\frac{-\sqrt{2} s}{2(s+c)} & -\frac{\sqrt{2}}{2} \\
1 & 0 \\
0 & 1 \\
\frac{-\sqrt{2}}{2(s+c)} & 0 \\
\frac{-\sqrt{2}}{2(s+c)} & 0
\end{array}\right]
$$

Matrix $\widetilde{\boldsymbol{T}}$ can be calculated by making use of the auxiliary schemes reported in Fig. (6). It takes the value

$$
\widetilde{\boldsymbol{T}}=\left[\begin{array}{clllll}
1 & 0 & 0 & 0 & 0 & 0 \\
0 & 1 & 0 & 0 & 0 & 0 \\
\frac{s^{2}}{s^{2}-c^{2}} & \frac{s c}{s^{2}-c^{2}} & 0 & 0 & \frac{s}{s^{2}-c^{2}} & \frac{c}{s^{2}-c^{2}} \\
\frac{s c}{s^{2}-c^{2}} & \frac{s^{2}}{s^{2}-c^{2}} & 0 & 0 & \frac{c}{s^{2}-c^{2}} & \frac{s}{s^{2}-c^{2}}
\end{array}\right]
$$

In order to calculate (16) it is necessary to add the expression of the diagonal element of the flexibility matrix $\overline{\boldsymbol{F}}$ corresponding to the $i$-th bar

$\overline{F_{l}}=\frac{\ell_{i}}{E A+t_{i}}$

Assuming the known the displacements collected in $\dot{\boldsymbol{u}}$ the solution of (16) can be achieved in the following two steps

$\widetilde{\boldsymbol{T}} \boldsymbol{y}=\dot{\boldsymbol{u}}$

$$
\overline{\boldsymbol{F}} \boldsymbol{t}=\boldsymbol{y}
$$

This is necessary since the problem defined by (16) is nonlinear in the unknowns collected in $\boldsymbol{x}$ and also convenient for the particular structure of matrix $\overline{\boldsymbol{F}}$.

For the first system of (51), simple calculation provides $y_{1}=\dot{u}_{2} y_{2}=\dot{v}_{3}$

$$
y_{5}=\frac{\left(\dot{u}_{4}\left(s^{2}-c^{2}\right)-\dot{u}_{2} s^{2}+\dot{v}_{3} s c\right) s+\left(\begin{array}{c}
\dot{u}_{4}\left(s^{2}-c^{2}\right)+ \\
\dot{u}_{2} s c-\dot{v}_{3} s^{2}
\end{array}\right) c}{s^{2}-c^{2}}
$$

while there are no conditions on $y_{3}$ and $y_{4}$ The particular structure of $\overline{\boldsymbol{F}}$ permits the following rearrangement

$$
\begin{aligned}
& {\left[\begin{array}{cccc}
y_{1} S_{11} & y_{1} S_{21} & -\ell_{1} S_{11} & -\ell_{1} S_{21} \\
y_{2} S_{12} & y_{2} S_{22} & -\ell_{2} S_{12} & -\ell_{2} S_{22} \\
y_{5} S_{15} & 0 & -\ell_{5} S_{15} & 0 \\
y_{6} S_{16} & 0 & -\ell_{6} S_{16} & 0
\end{array}\right]\left[\begin{array}{l}
x_{1} \\
x_{2} \\
\dot{x}_{1} \\
\dot{x}_{2}
\end{array}\right]=} \\
& {\left[\begin{array}{l}
-y_{1} E A+\ell_{1} \dot{t}_{1}^{\prime} \\
-y_{2} E A+\ell_{2} \dot{t}_{2}^{\prime} \\
-y_{5} E A+\ell_{5} \dot{t}_{5}{ }^{\prime} \\
-y_{6} E A+\ell_{6} \dot{t}_{6}^{\prime}
\end{array}\right]}
\end{aligned}
$$

which, when solved, gives the unknowns

$$
\begin{gathered}
x_{1}=\frac{\ell_{6} S_{16}\left(y_{5} E A-\ell_{5} \dot{t}_{5}{ }^{\prime}\right)+\ell_{5} S_{15}\left(-y_{6} E A+\ell_{6} \dot{t}_{6}{ }^{\prime}\right)}{\left(\ell_{5} y_{6}-\ell_{6} y_{5}\right) S_{15} S_{16}} \\
\dot{x}_{1}=\frac{y_{5} S_{15}\left(-y_{6} E A+\ell_{6} \dot{t}_{6}{ }^{\prime}\right)-y_{6} S_{16}\left(-y_{5} E A+\ell_{5} \dot{t}_{5}{ }^{\prime}\right)}{\left(\ell_{5} y_{6}-\ell_{6} y_{5}\right) S_{15} S_{16}} \\
x_{2}=\frac{\ell_{2} S_{22} \bar{b}_{1}+\ell_{1} S_{21} \bar{b}_{2}}{\left(\ell_{1} y_{2}-\ell_{2} y_{1}\right) S_{21} S_{22}} \\
\dot{x}_{2}=\frac{y_{1} S_{21} \bar{b}_{2}-y_{2} S_{22} \bar{b}_{2}}{\left(\ell_{1} y_{2}-\ell_{2} y_{1}\right) S_{21} S_{22}}
\end{gathered}
$$

where the following positions are used 
$\bar{b}_{1}=-y_{1} E A+\ell_{1} \dot{t}_{1}^{\prime}-\left(y_{1} x_{1}-\ell_{1} \dot{x}_{1}\right) S_{11}$

$$
\bar{b}_{2}=-y_{2} E A+\ell_{2} \dot{t}_{2}^{\prime}-\left(y_{2} x_{1}-\ell_{2} \dot{x}_{1}\right) S_{12}
$$

As in the examples illustrated above, in order to compare the obtained results it is necessary to estimate the nodal displacements collected in $\dot{\boldsymbol{u}}$. This can be done by evaluating the displacements of the scheme reported in Fig. (4c) caused by the forces $\dot{f}_{0}$ and $\dot{f}_{v}$.

By referring to the current configuration and in the framework of the flexibility method, axial force increments on the 3rd and 4th bar, see (8), can be computed from the following system

$$
\left[\begin{array}{cc}
\sqrt{2}+\frac{1+s^{2}}{(s+c)^{2}} & \frac{s}{(s+c)} \\
\frac{1+s^{2}}{(s+c)^{2}} & 1+\sqrt{2}
\end{array}\right]\left[\begin{array}{l}
\dot{t}_{3} \\
\dot{t}_{4}
\end{array}\right]=\frac{\sqrt{2}}{2}\left(\dot{f}_{o}+\dot{f}_{v}\right)\left[\begin{array}{c}
\frac{1+s^{2}}{(s+c)^{2}} \\
\frac{s}{s+c}
\end{array}\right]
$$

that when it is solved it produces

$\left[\begin{array}{c}\dot{t}_{3} \\ \dot{t}_{4}\end{array}\right]=\frac{\dot{f}_{o}+\dot{f}_{v}}{1+\sqrt{2}\left(1+s^{2}\right)+(\sqrt{2}+2)(s+c)^{2}}\left[\begin{array}{c}\frac{\sqrt{2}}{2}+\left(1+s^{2}\right) \\ s(s+c)\end{array}\right]$

from which, using (5), $\dot{\boldsymbol{t}}$ can be calculated. Successively, the requested displacements can be calculated by means of virtual work (9).

Results deriving from the proposed identification approach depends again only on the $a / \ell$ ratio. In the range $\frac{a}{\ell}=2 \times 10^{-7} \cdots 2 \times 10^{-4}$ it varies in a practically linear fashion from $2.23 \times 10^{-7} \ldots 2.23 \times 10^{-4}$.

It is also interesting to study how the proposed identification approach is affected by the errors that certainly exist in the considered identification problem. For example, nodal displacements are obtained from measurements and thus are, certainly, affected by errors. In order to simply evaluate the effects of measurement errors, we indicate with $w$ and $\widetilde{w}$ the real and the measured displacements respectively. In this case the data error parameter $\xi$ can implicitly be defined as $\widetilde{w}=(1+\xi) w$

Table 2 reports for $a / \ell=0.0005$ the identification error increasing data error parameter $\xi$. In addition to data reported in the beginning of this section we have assumed $\ell=5$ $\mathrm{m}, \dot{f}_{o}=1000 \mathrm{~N}$ and $\dot{f}_{v}=100 \mathrm{~N}$.

These results show, in an unambiguous way, the necessity of special techniques to filter the errors that are always present in the data. A filtering strategy such as that proposed by Tikhonov [15] could be useful to solve actual problem.

\section{CONCLUDING REMARKS}

This paper has sketched an identification procedure able to reconstruct actual axial forces on a pin-jointed truss. The proposed procedure is based on the results gathered through a simple mechanical test that uses a prescribed load and the measured displacement induced. In the framework of the flexibility method, the link between the axial forces to be identified and the structural response to the test load is described in the context of standard linear elasticity.

In Section 3 simple numerical tests showed the main characteristics of the proposed procedure and also highlighted its performances. When measurement errors are taken into account, a quick view of the identification of axial forces suggests that an ad hoc procedure is strongly necessary to filter such errors as it is usual in the identification procedures.

Table 2. Six-Bar Truss Problem: Identification Error $\eta$ Varying Data Error Parameter $\xi$ for $a / \ell=0.0005$.

\begin{tabular}{|c|c|c|c|c|}
\hline$\xi$ & $10^{-7}$ & $10^{-6}$ & $10^{-5}$ & $10^{-4}$ \\
\hline$\eta$ & $3.90 \times 10^{-7}$ & $1.52 \times 10^{-3}$ & $1.95 \times 10^{-2}$ & $2.00 \times 10^{-1}$ \\
\hline
\end{tabular}

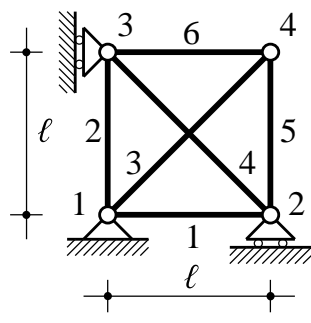

(a)

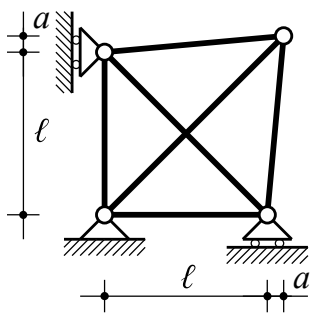

(b)

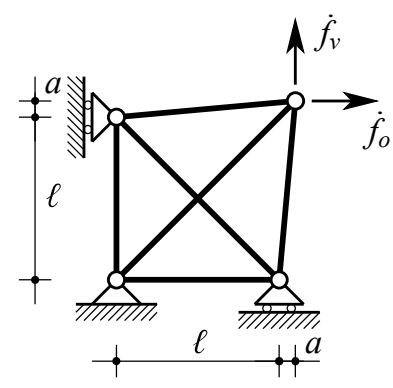

(c)

Fig. (4). Six-bar truss problem: stress-free configuration (a), current configuration (b) and test load (c).

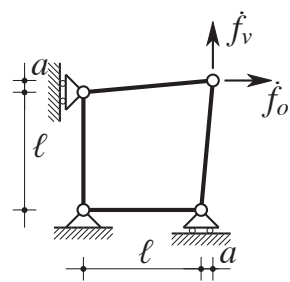

(a)

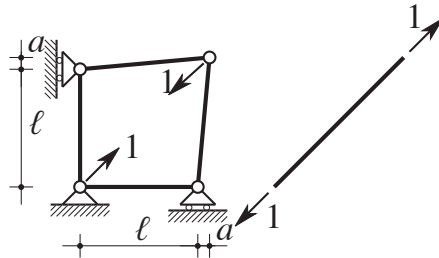

(b)

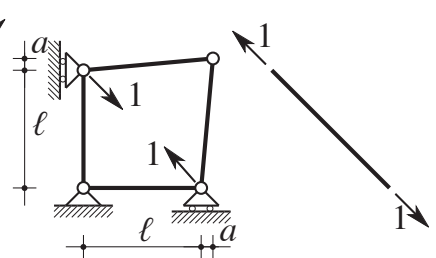

(c)

Fig. (5). Six-bar truss problem: auxiliary schemes for the calculations of (t) and (s) 


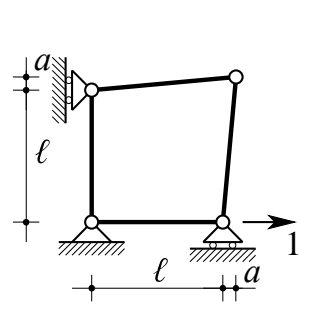

(a)

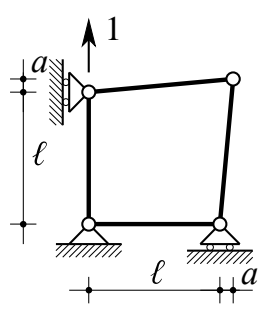

(b)

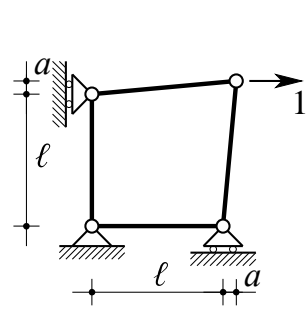

(c)

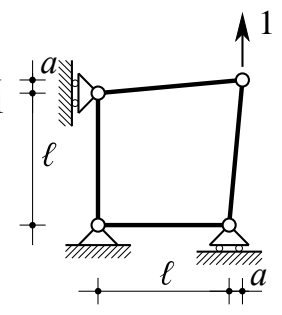

(d)

Fig. (6). Six-bar truss problem: auxiliary schemes for the calculation of matrix $\widetilde{\boldsymbol{T}}$.

Some future developments are synthetically listed below: 1. In this work we have considered only statically indeterminate and kinematically determined pin-jointed trusses, it appears interesting to extend this approach to prestressed mechanisms.

2. Only simple problems were considered here. Problems of medium and large dimensions, specially from the technical point of view, require particular care and specific strategies to be computationally efficient.

3. Although the flexibility method appears to be more promising as framework to identify the stress-state, the capabilities of the stiffness method should be adequately explored.

4. Implementations of more sophisticated constitutive laws as briefly described in Section 2, have to be explored to understand computational costs and advantages.

5. Pin-jointed trusses were considered important due to their technical applications and also for the simplicity of the mathematical model which used to study them. Viewing the procedure presented here in a finite element framework, a small effort would be adequate for the natural extension of the results presented here.

6. As it is well known, the solution of an identification problem necessarily requires specific strategies capable of filtering errors which cannot be eliminated, e.g. those on the data. Such errors occur from various causes for e.g. inaccuracy of the data, approximation of the mathematical model and sometimes due to the numerical model and rough accuracy of the solution algorithm. Although there are numerous general purpose strategies that can be used to reduce the aforementioned errors but a specifically designed procedure has its own benefits as it can deal with specific problem.

7. Finally, it would be interesting to explore the results obtained from a geometrically nonlinear model.

\section{ACKNOWLEDGEMENTS}

None declared.

\section{REFERENCES}

[1] Y. C. Fung, A First Course in Continuum Mechanics, PrenticeHall: USA, 1994.

[2] A. Hoger, "On the determination of residual stress in an elastic body", Journal of Elasticity, vol. 16, pp. 303-324, 1986.

[3] B. C. P. Burke, S. O. Kim and K. S. Kim, "Partial polar decomposition inverse method applied to determination of internal stresses in an elastic complex structures", International Journal of Solids and Structures, vol. 44, pp.2010-2020, 2007.

[4] F. Zhang, A. J. Kassab and D. W. Nicholson, "A boundary element solution of an inverse elasticity problem and application to determining residual stress and contact stress", International Journal of Solids and Structures, vol. 34, no. 16, pp. 2073-2086, 1997.

[5] H. D. Bui, Inverse Problems in the Mechanics of Materials: An Introduction, CRC Press: USA, 1994.

[6] A. Puro,"Magnetophotoelasticity as parametric tensor field tomography", Inverse Problems, vol. 14, pp. 1315-1330, 1998.

[7] R. L. Robertson, "Boundary identifiability of residual stress via the Dirichlet to Neumann map", Inverse Problems, vol. 13, pp. 11071119, 1997.

[8] C. S. Man, "Hartig's law and linear elasticity with initial stress", Inverse Problems, vol. 14, pp. 313-319, 1998.

[9] R. L. Robertson, "Determining residual stress from boundary measurements", Journal of Elasticity, vol. 52, pp. 63-73, 1998.

[10] G. E. B. Tan and S. Pellegrino, "Non-linear dynamic identification: An application to prestressed cable structures", Journal of Sound and Vibration, vol. 208, no. 1, pp. 33-45, 1997.

[11] S.Pellegrino, "Analysis of prestressed mechanism", International Journal of Solids and Structures, vol.26. no. 12, 1329-1350, 1990.

[12] S.Guest, "The stiffness of prestressed frameworks: A unifying approach", International Journal of Solids and Structures, vol. 43, pp. 842-854, 2006

[13] R. K. Livesley, Matrix method of structural analysis, $2^{\text {nd }}$ ed. Pergamon Press: Oxford, 1975.

[14] J. F. Bell, The experimental foundations of solid mechanichs, vol. I, Springer-Verlag: Berlin, 1973.

[15] A. N. Tikhonov and V. Y. Arsenin, Solution of ill-posed problems, John Wiley \& Sons: New York, 1977.

\section{CONFLICT OF INTEREST}

The authors confirm that this article content has no conflicts of interest. 